\title{
5
}

\section{How right is wrong}

M. Schlumberger

Cap Gemini Innovation

Les Jardins de Maupertuis

Bat. Karelian - 7 Chemin de la Dhuy

38240 Meylan, France

Tel +3376764777 Fax +3376 764748

e-mail mschlumb\%cgsmail@capgemini.fr

\begin{abstract}
Experience shows that in many cases it is better to reach the "wrong " decision rather than wait for the " right " one, specially when there exist mechanisms to monitor the outcome of the decision and support the improvement of both this outcome and the decision making process itself This paper shows pracical examples fom various sources to develop this theme.
\end{abstract}

\section{Keywords}

East Europe, decision making, reporting and monitoring, process improvement, changing short term mentalities.

\section{INTRODUCTION}

This paper is based on experiences both personal and reported. It came to being written after the "collapse" of the so-called socialist systems in Eastern Europe. Having had the opportunity to work there with decision making experts, it became evident that only some parts of the system needed improvement, in my mind mostly because the system insisted on reaching the right decision only. Many similar situations that could greatly benefit from allowing for errors can be found elsewhere, including of course in Western Europe.

- In the first section this paper surveys some of the advantages of reaching the "right" decision in a given situation, as well as gives a few caveats to many reward systems that are associated with this approach.

- The second section develops the reporting and monitoring issues, showing that these have in fact a profound pavlovian impact on how people behave in day to day situations. 
- The third section builds the central idea of the paper, that nothing is ever always right, and that it generally is better to be able to cope with wrong decisions than to impose to (and bear with) be always right.

- The next section focuses on the improvement issues, of the decisions themselves, but also of the processes used to reach those decisions.

- This is followed by a conclusion summing up the main points and messages, as well as indicating points of further interest.

A decision here is considered as more than just having an idea. A decision has some visible (big or small) effects on the world at large.

\section{MAKING THE RIGHT DECISION}

Many, if not most cultures take pride in insisting that it is essential to do one's utmost to reach the "right" decision. Hence possibly the flourish of the so-called "Decision Support Systems", be they anywhere from simple Information Systems, to fancy Organization Transformation Support Systems (Humphreys, 1989).

Reaching this "right" decision can be a matter of life and death in many cases, such as driving one's car or reaching a medical diagnosis. Many school tests are examples where the difference between right and wrong can be quite expensive, in time, in money, in opportunities. Hence we are expected to take to heart finding this "right decision". Yet costs associated with achieving this proper choice may be out of balance in view of the expected results, and there are cases, at least in the Western culture, where short-cuts can be accepted and are at times encouraged.

For instance, a well known (to students) statistical fluke in a many multiplechoice tests, is that one is often better off to randomly answer questions, hence get an average score, rather than not answer them, or, of course, always answering wrong.

\section{Absolutely right or right now}

Another example is deeply linked to the culture, where one is - or isn't - expected to answer a question quickly rather than accurately. This difference is specially visible when an American is faced with an Arab, East Asian or a Soviet environment where reaching the right decision is more important (for various reasons, such as not loosing face) than reaching any decision fast.

Western Culture, such as the "typical" American one, strongly pushes people to reach a decision quickly, and accepts in many cases that this decision may be flawed in the middle to long term (Phillips, 1992; Humphreys and Nappelbaum, 1997). It is considered normal in this sense for laws (and even "constitutions") to change, and for politicians to modify their position on issues. It is accepted that other 
professions can also make "mistakes", and there are many instances and methods to correct these.

Driving is a case we all know, where when you reach a fork in the road, you must choose a turn. If you keep the course, while deciding on the best option, you crash.

This paper tries to explain the consequences of this difference in attitude towards reaching a decision on organisations. Most of this difference does not seem to be ingrained, but rather probably comes from the global organisational attitudes towards failure, imposed, or reinforced within organisations, by the reporting and monitoring schemes. A similar attitude can be observed in organisations undergoing change, as shown elsewhere (Schlumberger, 1994).

\section{REPORTING AND MONITORING}

A previous paper (Schlumberger 1994) has shown that reporting and monitoring within an organisation reinforce specific attitudes in people reporting their results and being monitored. The Hawthorne experiments already showed this in the late 1920s (Roethlisberger and Dickson, 1939), with the often told conclusion:

\section{you improve what you measure (or at least pay attention to)}

When setting up reporting schemes, organisations should decide whether they want their stake-holders to reach the right decision, or simply to reach a reasonable one, the difference having far-reaching consequences both on the behaviour of the people concerned and on that of the organisation, internally and externally. Most organisations rarely if ever make this choice consciously, and obviously they do not realise the full consequences of such a choice.

In many cases, we are faced with the «street light syndrome' " where reporting focuses on easy to obtain figures rather than on relevant ones. Reporting sales is relatively easy, but a company lives on margins not on sales. Determination of real margins is more difficult, and less often reported!

This occurs much more frequently than one would expect, with as a consequence that people focus on getting good figures for reporting rather than on getting the deed done as well as possible. One old but still valid example is that of the « Great

\footnotetext{
'So, late one evening you meet this drunkard kneeling in the gutter, under a street lamp, obviously looking for something:

- What are you doing there?

- Oh I'm looking for my car key, I lost it when I locked my car.

- But where is your car? I don't see it, why don't you look next to your car?

- My car is down there, but there is no bloody light nest to the car!
} 
leap forward " in China, where one of the success measure was the gross production of pig iron, which did, in deed boost, but that came to nothing, in part because of the very low quality of the results.

Another example is linked to the measure of "productivity " most often used for software: KLODC (line of code delivered per day per person), which pleads for the insertion of neutral lines, safe, won't do any harm, but will boost the "productivity" figure.

Pavlov conducted his most famous experiments on dogs, but his conclusions apply to people in organisations, in ways that both the organisation and the concermed individuals are usually unaware of (Homans, 1961). The measurements used in organisations push people to do what is needed in order to improve the readings, pretty much independently of the ultimate benefits incurred.

The next section shows the importance of focusing on the ultimate goals of the organisation, hence on accepting that issues may be at a given time monitored negatively, yet keeping an environment where some mistakes can be accepted socially!

\section{MAKING A WRONG DECISION}

In all cases, a "wrong" decision is not as "good" as a "right" one, so why argue about this? The answer is quite simple and lies both in the time it takes to reach a decision and on the real consequences of not having any decision, or worse, any one willing to make a decision, and to take responsibility.

The example of reaching a fork in the road is a simple one that reinforces the message. The faster you drive, the faster you have to decide, in order to survive. A «bad» decision (taking the wrong turn) is much better than no decision (crashing the car).

Giving someone responsibility for something implies allowing, accepting that this person makes mistakes on the issue. This is in fact much more than a jest. It really should mean (although we shall soon see it does not always) what it says, i.e. when someone is responsible, this person can also make mistakes, this is part of the assignment!

Most organisations, be they social or business oriented, recognise that people "naturally" make mistakes. Suffice it to see the importance given to atoning for one's sins in most if not all religions, i.e. these religions have placed a process whereby they accept the mistakes (in their definition) made by people and give a 
way to recover from these. Religions that do not forgive or allow people somehow to recover from their sins have feiw followers.

Reaching the "wrong" decision is a common occurrence while the consequences of this decision depend in part on the immediate environment where this decision was reached, and in part on the way its consequences are treated later on. A decision that is deemed « wrong » at a given time can turn into the right one later!

Before going any further in this discussion, one should be fully aware that "no decision" is, in all cases, a decision, i.e. keeping the current orientations has its own set of consequences. For many people not rocking the boat is no decision, and is easier than any kind of decision.

Sailing is another example, where when a craft is going towards some danger, no decision is in practice a very dangerous course to follow!

A common misconception is that you can delay making a decision. In fact, in most cases, this simply prolongs the status quo, which, in itself, already is a decision, with its usual set of consequences. Not making a decision is making one! One way around this issue is to have a regular change of direction by default, which forces the issue of keeping the current track.

This is for instance what is done in modern freeway design, where there are no long straight lines, but regular wide curves, that force the driver to stay alert and in active control of her car.

Yet many environments, not least the educational one, strongly push for only reaching the « right » decision, and avoiding an answer to a question rather than giving the "wrong" one, which does not prepare for real life situations.

Time is more and more " of the essence ", both because there is less time available to reach a decision (think of the driving or sailing analogies above), but also because, as the environment evolves faster and faster, the right decision under today's conditions has a good chance of being the wrong one for tomorrow.

A simple consequence of this continuous change is that the longer you ponder your decision, the more chances there are that it will not be right when you reach it, as external circumstances will have changed by then. Many decision making and decision analysis practitioners have had to come to terms with this!

In a fast changing environment, you do much better with a reasonable decision taken for the short term (which could known to be bad in the long term), along with some monitoring system in order to make sure that whatever needs to be done to keep up with the environmental modifications, is done, rather than sticking with some long term choice that will most presumably start and stay bad.

This pushes for a reinforced dichotomy between the means and the end, whereby the immediate decisions reflect on the means to be taken now, that must change in 
time, and corrected when necessary, while the end, the purpose, the objective stays constant over a longer period.

Responsibility for achieving a specific end must be given relatively independently of the means used to reach this end (within ethical and resource bounds of course). Overall responsibility for reaching a goal implies acceptance and support from the hierarchy of the (possibly) « wrong » decisions taken to reach it. People are to be judged on results before being judged on means, even if it is much easier to evaluate the current means spent than the end to come. Responsibility is also a matter of trust.

handing over responsibility implies acceptance of mistakes

\section{IMPROVEMENT OF THE RESULTS AND THE PROCESS}

"Wrong" decisions are common. If people are restricted to "right" decisions, they naturally avoid taking decisions and responsibilities as much as possible, as this avoidance entails fewer personal risks, and personal consequences than taking a "wrong" decision.

Rather than limiting people to reaching the "right" decision, hence leading to decision avoidance, with all of its consequences (driving has been used as an example, but politics is another well described one), it is better to accept the fact that « wrong " decisions are made, and to have the organisation cope with this.

The two main aspects of coping can be described as:

- Improvement by eventual change of the decisions, in view of its results, and

- Definition and improvement of a full decision making process that monitors decisions and their results, in order to allow for the improvement of the results.

These two aspects are further described below.

\section{Improvement of results}

The issue here is to accept decisions, whatever they are, and to focus on improving their results.

"You improve what you measure" could be a conclusion of the Hawthorne set of experiments, or a re-wording of a famous quote from Lord Rutherford.

This is so true that, for instance in software development, measuring the results in terms of lines of code produces... mostly lines of code, that as often as not have little relevance to the problem to be solved (Jeffery and Lawrence, 1979)!

Organizations that want to improve the results of decisions must monitor these results. The earlier the consequences of a «bad» decision are found, the more one 
can do about it. Yet some of the reluctance shown towards accepting bad decisions, may be linked to the reluctance of some organisations to monitor their own effectiveness in an objective way.

A classical case has been that of governments trying to lower unemployment rates (instead of unemployment). When unemployment stays at high levels, two common solutions are first to change the definition of unemployment, generally lowering the "official" rate, or second, to ignore the results, describing these as irrelevant to the current economic or whatever situation.

Once results are properly monitored, and only very few organisations actually seem do so in serious terms (there is a legal obligation to monitor the financial results, mostly for tax purposes though, which is not always the best incentive), can these organisations start to monitor the influence of decisions and work on improvements.

Despite the trend to play ostrich, most organisations have found that monitoring improves results, and insist on reporting schemes that could allow them to find, fast and effectively, what is actually going on. Yet, given the flood of data streaming in, there is a chance that most of it is simply ignored, unless some automatic monitoring system is there that at least reports grossly out of line results.

In all cases, improving the result implies that challenging the current course followed (after a decision) is an accepted fact of the social life of the organisation. Most organisations do not accept such challenges, as they can wreak havoc with their internal workings. Yet consensus-based organisations have a much easier time to challenge continually the course followed than do authoritarian organisations. Without accepting this continuous challenge, any improvement is very difficult.

Improving the result normally implies, beyond measuring the actual result you want to improve, improving the process that reaches this result. Indeed, as mentioned above, in most cases, current processes do not condone, or even accept "bad" results, hence must be improved before this can be done. This process improvement is the topic of the next section.

\section{Improvement of process}

In order to allow for "bad" decisions, and to be able to improve on them, most organisations must improve their current decision making and evaluating process, and all must continually improve this process. Yet, the current process typically the result of such a "bad" set of decisions! This section elaborates on this process improvement issue, by giving the general guidelines of such an improvement and the sort of process resulting from such an effort. 
The Software Engineering Institute (SEI) in Pittsburgh at the instigation of the US Department of Defence, which wanted to improve the quality of the software it outsourced, has formalised five levels of Software Development Process in an organisation, that can pretty much be applied to any decision making process (Humphrey, 1988). Reworded, these are:

- Nothing: there is no such process known

- Aware: There is a process, somewhere,

- Repeatable: a process can be done a second time around

- Defined: a process is known and explainable

- Optimised: the process is continuously measured and improved upon.

Practical experience has shown that you can go from your current level up one at a time, no more. Experience also has led SEI to refine its definitions and assessment methods, as well as focal points that help an organisation going from one stage to the next.

This change in the SEI model reinforces the point of the paper: the issue is not to have the right answer, but an answer that is confronted to reality and improved accordingly. Improving an organisational process thus requires first the awareness that such a process exists, and then at least an attempt at the understanding and formalisation of the process. Once this is formalised (and the use of computer based process management tools is pushing a number of organisations in this direction), improvements can be thought and tried out.

In the same line of improvement, one must recognise that the current situation depends on past decisions (both active and passive), and that, in order to judge the validity of these previous decisions, one must know which ones are currently at stake. Keep a log of decisions (active and passive ones) and reasons for those.

Then, if the judgement to be applied is a commercial (or say a political) one, decisions should be judged on this measure, and no other. There are no "good" or "bad" decisions, in commercial terms (or political) there are "beneficial" or "expensive" ones, and each can. be measured. In order to improve the decision process, one should at least try to find the "objective" causes for costly decisions, these are usually linked to the environment, not to the people who "decide".

Organizations, as well as people within them have to accept socially and psychologically that decisions often are flawed, and that this should not systematically bear on decision makers. The process improvement is concerned with improving the chances of reaching good decisions (as compared to always reaching the right decisions), as well as minimising the consequences of a bad one in the short and long term.

The decision " $\log$ » will help in many ways: it will explain why a given decision was reached, and will force the next decision makers to consciously choose to approve or disapprove it on valid grounds. It will also serve as a basis for 
formulating the decision process and eventually improving it, again on a sound basis.

\section{CONCLUSION}

This paper has shown how the organisational pressure to always be right is both unrealistic in its expectations and in it consequences: people do and will continue to make mistakes, and when pushed not to make them, they will abstain from any initiative. Organisations should focus instead on accepting this foible and improving both the decision making process and the uptake of decision. A few salient points have been made, to be used by organisations that are intent on improving their attitude towards decision making:

- Accept "wrong decisions", and work with, instead of against them: monitor them and improve (if given a chance), not changing the current course is such a decision.

- Improve process to accept these, evaluate results, and make certain things improve

- Focus on difficulties in changing the short term mentalities in evaluation of results, this will make it easier to progress towards set goals that reinforce the strength/purposes of the organisation.

\section{ACKNOWLEDGEMENTS}

Acknowledgements are due to Patrick, Zita and Feco.

\section{REFERENCES}

Homans, G. (1961) Social behaviour: Its elementary forms. Harcourt Brace, New York.

Humphrey, W. S. (1988) Characterising the software process: A maturity framework. IEEE Software, 5, 73-79

Humphreys, P. C. (1989) Intelligence in decision support, in Knowledge based management support systems (eds. G. Doukidis, F. Land and G. Miller), Ellis Horwood, Chichester.

Humphreys, P. C. and Nappelbaum, E. L. (1997) Structure and communications in the process of organisational change: Eastern European experience and its general relevance. This volume 
Jeffery, D. R. and Lawrence, M. J. (1979) An inter-organisational comparison of programming productivity, in Proceedings of the 4th ICSE, Munich, pp. 369377, IEEE Catalogue No. 79 CH1479-5C

Phillips, L. D. (1992) Gaining corporate commitment to change, in Executive information systems and decision support (ed. C. Holtham) Chapman and Hall, London.

Roethlisberger F. J. and Dickson, W. J. (1939) Management and the worker. Harvard University Press, Cambridge, Mass.

Schlumberger, M (1994). Project Management considered as an impediment to change. Proceedings of the IFIP WG8.3 conference on Decision Support and Organizational Transformation, San Sebastian, Spain.

\section{BIOGRAPHY}

Maurice Schlumberger is Scientific Director of Cap Gemini Innovation, scientific research centre of Cap Gemini. He has directed many projects on software development, and information systems and project management systems development.. 